\title{
The stability of local strong solutions for a shallow water equation
}

\author{
Shaoyong Lai", Haibo Yan, Hongjing Chen and Yang Wang
}

"Correspondence:

Laishaoy@swufe.edu.cn

Department of Mathematics,

Southwestern University of Finance

and Economics, Chengdu, 611130,

China

\begin{abstract}
We establish the $L^{1}$ stability of local strong solutions for a shallow water equation which includes the Degasperis-Procesi equation provided that its initial value lies in the Sobolev space $H^{5}(R)$ with $s>\frac{3}{2}$. The key element in our analysis is that the $L^{\infty}$ norm of the solutions keeps finite for all finite time $t$.
\end{abstract}

MSC: 35G25; 35L05

Keywords: $L^{1}$ stability; strong solutions; shallow water equation

\section{Introduction}

From the propagation of shallow water waves over a flat bed, Constantin and Lannes [1] derived the equation

$$
g_{t}+g_{x}+\frac{3}{2} \rho g g_{x}+\mu\left(\alpha g_{x x x}+\beta g_{t x x}\right)=\rho \mu\left(\gamma g_{x} g_{x x}+\delta g g_{x x x}\right),
$$

where the constants $\alpha, \beta, \gamma, \delta, \rho$ and $\mu$ satisfy certain restrictions. As illustrated in [1], using suitable mathematical transformations turns Eq. (1) into the form

$$
g_{t}-g_{t x x}+k g_{x}+m g g_{x}=a g_{x} g_{x x}+b g g_{x x x},
$$

where $a, b, k$ and $m$ are constants. We know that the Camassa-Holm and DegasperisProcesi models are special cases of Eq. (2). Lai and Wu [2] established the well-posedness of local strong solutions and obtained the existence of local weak solutions for Eq. (2).

The aim of this paper is to investigate a special case of Eq. (2). Namely, we study the shallow water equation

$$
g_{t}-g_{t x x}+k g_{x}+m g g_{x}=3 g_{x} g_{x x}+g g_{x x x}
$$

where $k \geq 0$ and $m>0$ are constants. Letting $y=g-\partial_{x x}^{2} g, v=\left(m-\partial_{x x}^{2}\right)^{-1} g$ and using Eq. (3), we derive the conservation law

$$
\int_{R} y v d x=\int_{R} \frac{1+\xi^{2}}{m+\xi^{2}}|\hat{g}(t, \xi)|^{2} d \xi=\int_{R} \frac{1+\xi^{2}}{m+\xi^{2}}\left|\hat{g}_{0}(\xi)\right|^{2} d \xi \sim\left\|g_{0}\right\|_{L^{2}(R)},
$$

○2014 Lai et al.; licensee Springer. This is an Open Access article distributed under the terms of the Creative Commons Attribution License (http://creativecommons.org/licenses/by/2.0), which permits unrestricted use, distribution, and reproduction in any medium, provided the original work is properly cited. 
where $g_{0}=g(0, x)$ and $\hat{g}(t, \xi)$ is the Fourier transform of $g(t, x)$ with respect to variable $x$. In fact, the conservation law (4) plays an important role in our further investigations of Eq. (3).

For $m=4, k=0$, Eq. (3) reduces to the Degasperis-Procesi equation [3]

$$
g_{t}-g_{t x x}+4 g g_{x}=3 g_{x} g_{x x}+g g_{x x x}
$$

Various dynamic properties for Eq. (5) have been acquired by many scholars. Escher et al. [4] and Yin [5] studied the global weak solutions and blow-up structures for Eq. (5), while the blow-up structure for a generalized periodic Degasperis-Procesi equation was obtained in [6]. Lin and Liu [7] established the stability of peakons for Eq. (5) under certain assumptions on the initial value. For other dynamic properties of the Degasperis-Procesi (5) and other shallow water models, the reader is referred to [8-19] and the references therein.

The objective of this work is to establish the $L^{1}(R)$ stability of local strong solutions for the generalized Degasperis-Procesi equation (3) under the condition that we let the initial value $g_{0}$ belong to the space $H^{s}(R)$ with $s>\frac{3}{2}$. Here we address that the $L^{1}$ stability of local strong solutions for Eq. (3) has never been established in the literature. Our main approaches come from those presented in [20].

This paper is organized as follows. Section 2 gives several lemmas. The main result and its proof are presented in Section 3.

\section{Several lemmas}

The Cauchy problem of Eq. (3) is written in the form

$$
\left\{\begin{array}{l}
g_{t}-g_{t x x}+k g_{x}+m g g_{x}=3 g_{x} g_{x x}+g g_{x x x}, \\
g(0, x)=g_{0}(x)
\end{array}\right.
$$

which is equivalent to

$$
\left\{\begin{array}{l}
g_{t}+g g_{x}+k \Lambda^{-2} g_{x}+\frac{m-1}{2} \Lambda^{-2}\left(g^{2}\right)_{x}=0 \\
g(0, x)=g_{0}(x)
\end{array}\right.
$$

where $\Lambda^{-2} f=\frac{1}{2} \int_{R} e^{-|x-y|} f d y$ for any $f \in L^{2}(R)$ or $L^{\infty}(R)$.

Let $Q_{g}(t, x)=\frac{m-1}{2} \Lambda^{-2}\left(g^{2}\right)+k \Lambda^{-2} g$ and $J_{g}=\partial_{x}\left(\frac{m-1}{2} \Lambda^{-2}\left(g^{2}\right)+k \Lambda^{-2} g\right)$, we have

$$
g_{t}+\frac{1}{2}\left(g^{2}\right)_{x}+J_{g}=0
$$

Lemma 2.1 For problem (6) with $m>0$, it holds that

$$
\int_{R} y v d x=\int_{R} \frac{1+\xi^{2}}{m+\xi^{2}}|\hat{g}(t, \xi)|^{2} d \xi=\int_{R} \frac{1+\xi^{2}}{m+\xi^{2}}\left|\hat{g}_{0}(\xi)\right|^{2} d \xi \sim\left\|g_{0}\right\|_{L^{2}(R)} .
$$

In addition, there exist two positive constants $c_{1}$ and $c_{2}$ depending only on $m$ such that

$$
c_{1}\left\|g_{0}\right\|_{L^{2}(R)} \leq c_{1}\|g\|_{L^{2}(R)} \leq c_{2}\left\|g_{0}\right\|_{L^{2}(R)} .
$$


Proof Letting $y=g-\partial_{x x}^{2} g$ and $v=\left(m-\partial_{x x}^{2}\right)^{-1} g$ and using Eq. (3), we have $g=m v-\partial_{x x}^{2} v$ and

$$
\begin{aligned}
\frac{d}{d t} \int_{R} y v d x & =\int_{R} y_{t} v d x+\int_{R} y v_{t} d x=2 \int_{R} v y_{t} d x \\
& =2 \int_{R}\left[\left(-\frac{m}{2} g^{2}\right)_{x}+k g_{x}+\frac{1}{2} \partial_{x x x}^{3} g^{2}\right] v d x \\
& =2 \int_{R}\left[\left(-\frac{m}{2} g^{2}\right)_{x} v+k g_{x} v+\frac{1}{2} \partial_{x} g^{2} \partial_{x x}^{2} v\right] d x \\
& =\int_{R}\left[\left(-m g^{2}\right)_{x} v+k g_{x} v+\left(g^{2}\right)_{x}(m v-g)\right] d x \\
& =-\int_{R} g\left(g^{2}\right)_{x} d x+k \int_{R}\left(m v_{x}-v_{x x x}\right) v d x \\
& =k \int_{R} v_{x x} v_{x} d x \\
& =0,
\end{aligned}
$$

from which we complete the proof.

Lemma $2.2([2])$ If $g_{0} \in H^{s}(R)$ with $s>\frac{3}{2}$, there exist maximal $T=T\left(u_{0}\right)>0$ and a unique local strong solution $g(t, x)$ to problem (6) such that

$$
g(t, x) \in C\left([0, T) ; H^{s}(R)\right) C^{1}\left([0, T) ; H^{s-1}(R)\right) .
$$

Firstly, we study the differential equation

$$
\left\{\begin{array}{l}
p_{t}=g(t, p), \quad t \in[0, T) \\
p(0, x)=x
\end{array}\right.
$$

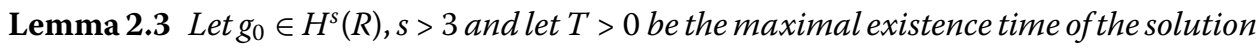
to problem (10). Then problem (10) has a unique solution $p \in C^{1}([0, T) \times R, R)$. Moreover, the map $p(t, \cdot)$ is an increasing diffeomorphism of $R$ with $p_{x}(t, x)>0$ for $(t, x) \in[0, T) \times R$.

Proof From Lemma 2.2, we have $g \in C^{1}\left([0, T) ; H^{s-1}(R)\right)$ and $H^{s-1}(R) \in C^{1}(R)$. Thus we conclude that both functions $g(t, x)$ and $g_{x}(t, x)$ are bounded, Lipschitz in space and $C^{1}$ in time. Using the existence and uniqueness theorem of ordinary differential equations derives that problem (10) has a unique solution $p \in C^{1}([0, T) \times R, R)$.

Differentiating (10) with respect to $x$ yields

$$
\left\{\begin{array}{l}
\frac{d}{d t} p_{x}=g_{x}(t, p) p_{x}, \quad t \in[0, T) \\
p_{x}(0, x)=1
\end{array}\right.
$$

which leads to

$$
p_{x}(t, x)=\exp \left(\int_{0}^{t} g_{x}(\tau, p(\tau, x)) d \tau\right) .
$$


For every $T^{\prime}<T$, using the Sobolev embedding theorem yields

$$
\sup _{(\tau, x) \in\left[0, T^{\prime}\right) \times R}\left|g_{x}(\tau, x)\right|<\infty
$$

It is inferred that there exists a constant $K_{0}>0$ such that $p_{x}(t, x) \geq e^{-K_{0} t}$ for $(t, x) \in$ $[0, T) \times R$. This completes the proof.

Lemma 2.4 Assume $g_{0} \in H^{s}(R)$ with $s>\frac{3}{2}$. Let $T$ be the maximal existence time of the solutiong to Eq. (3). Then we have

$$
\|g(t, x)\|_{L^{\infty}} \leq c_{0}\left\|g_{0}\right\|_{L^{2}}^{2} t+\left\|g_{0}\right\|_{L^{\infty}} \quad \forall t \in[0, T]
$$

where constant $c_{0}$ depends on $m, k$.

Proof Let $Z(x)=\frac{1}{2} e^{-|x|}$, we have $\left(1-\partial_{x}^{2}\right)^{-1} f=Z \star f$ for all $f \in L^{2}(R)$ and $g=Z \star y(t, x)$. Using a simple density argument presented in [6], it suffices to consider $s=3$ to prove this lemma. If $T$ is the maximal existence time of the solution $g$ to Eq. (3) with the initial value $g_{0} \in H^{3}(R)$ such that $g \in C\left([0, T), H^{3}(R)\right) \cap C^{1}\left([0, T), H^{2}(R)\right)$. From (7), we obtain

$$
g_{t}+g g_{x}=-(m-1) Z \star\left(g g_{x}\right)-k Z \star g_{x} .
$$

Since

$$
\begin{aligned}
-Z \star\left(g g_{x}\right) & =-\frac{1}{2} \int_{-\infty}^{\infty} e^{-|x-y|} g g_{y} d y \\
& =-\frac{1}{2} \int_{-\infty}^{x} e^{-x+y} g g_{y} d y-\frac{1}{2} \int_{x}^{+\infty} e^{x-y} g g_{y} d y \\
& =\frac{1}{4} \int_{\infty}^{x} e^{-|x-y|} g^{2} d y-\frac{1}{4} \int_{x}^{\infty} e^{-|x-y|} g^{2} d y
\end{aligned}
$$

and

$$
\begin{aligned}
\frac{d g(t, p(t, x))}{d t} & =g_{t}(t, p(t, x))+g_{x}(t, p(t, x)) \frac{d p(t, x)}{d t} \\
& =\left(g_{t}+g g_{x}\right)(t, p(t, x)),
\end{aligned}
$$

from (16), we have

$$
\frac{d g}{d t}=\frac{m-1}{4} \int_{-\infty}^{p(t, x)} e^{-|p(t, x)-y|} g^{2} d y-\frac{m-1}{4} \int_{p(t, x)}^{\infty} e^{-|p(t, x)-y|} g^{2} d y-k Z \star g_{x}
$$

from which we get

$$
\begin{aligned}
\left|\frac{d g(t, p(t, x))}{d t}\right| & \leq \frac{|m-1|}{4} \int_{-\infty}^{\infty} e^{-|p(t, x)-y|} g^{2} d y+\left|k Z \star g_{x}\right| \\
& \leq \frac{|m-1|}{4} \int_{-\infty}^{\infty} g^{2} d y+k\left|\int_{-\infty}^{\infty} \frac{1}{2} e^{-|x-y|} g_{y} d y\right|
\end{aligned}
$$




$$
\begin{aligned}
& \leq \frac{|m-1|}{4}\|g\|_{L^{2}}^{2}+k\|g\|_{L^{2}} \\
& \leq c\|g\|_{L^{2}(R)} \\
& \leq c\left\|g_{0}\right\|_{L^{2}(R)},
\end{aligned}
$$

where $c$ is a positive constant independent of $t$. Using (18) results in

$$
-c t\left\|g_{0}\right\|_{L^{2}(R)}+g_{0} \leq g(t, p(t, x)) \leq c t\left\|g_{0}\right\|_{L^{2}(R)}+g_{0} .
$$

Therefore,

$$
|g(t, p(t, x))| \leq\|g(t, p(t, x))\|_{L^{\infty}} \leq c t\left\|g_{0}\right\|_{L^{2}(R)}+\left\|g_{0}\right\|_{L^{\infty}} .
$$

Using the Sobolev embedding theorem to ensure the uniform boundedness of $g_{x}(s, \eta)$ for $(s, \eta) \in[0, t] \times R$ with $t \in\left[0, T^{\prime}\right)$, from Lemma 2.3 , for every $t \in\left[0, T^{\prime}\right)$, we get a constant $C(t)$ such that

$$
e^{-C(t)} \leq p_{x}(t, x) \leq e^{C(t)}, \quad x \in R .
$$

We deduce from the above equation that the function $p(t, \cdot)$ is strictly increasing on $R$ with $\lim _{x \rightarrow \pm \infty} p(t, x)= \pm \infty$ as long as $t \in\left[0, T^{\prime}\right)$. It follows from (20) that

$$
\|g(t, x)\|_{L^{\infty}}=\|g(t, p(t, x))\|_{L^{\infty}} \leq c t\left\|g_{0}\right\|_{L^{2}(R)}+\left\|g_{0}\right\|_{L^{\infty}}
$$

Lemma 2.5 Assume $g_{0} \in L^{2}(R)$. Then

$$
\left\|Q_{g}\right\|_{L^{\infty}\left(R_{+} \times R\right)},\left\|J_{g}\right\|_{L^{\infty}\left(R_{+} \times R\right)} \leq c_{0}\left\|g_{0}\right\|_{L^{2}}^{2},
$$

where $c_{0}$ is a constant independent of $t$.

Proof Using (7), we get

$$
\begin{aligned}
Q_{g}(t, x)= & \frac{m-1}{4} \int_{R} e^{-|x-y|} g^{2}(t, y) d y+\frac{k}{2} \int_{R} e^{-|x-y|} g d y, \\
J_{g}(t, x)= & \frac{m-1}{4} \int_{R} e^{-|x-y|} \operatorname{sign}(y-x) g^{2}(t, y) d y \\
& +\frac{k}{2} \int_{R} e^{-|x-y|} \operatorname{sign}(y-x) g(t, y) d y .
\end{aligned}
$$

It follows from (23)-(24) and Lemma 2.1 that (22) holds.

Lemma 2.6 Assume that $g_{1}(t, x)$ and $g_{2}(t, x)$ are two local strong solutions of equation (3) with initial data $g_{10}, g_{20} \in H^{s}(R), s>\frac{3}{2}$, respectively. Then, for any $f(t, x) \in C_{0}^{\infty}([0, \infty) \times R)$, it holds that

$$
\int_{-\infty}^{\infty}\left|J_{g_{1}}(t, x)-J_{g_{2}}(t, x)\right||f(t, x)| d x \leq c_{0}(1+t) \int_{-\infty}^{\infty}\left|g_{1}-g_{2}\right| d x
$$

where $c_{0}>0$ depends on $t, f,\left\|g_{10}\right\|_{L^{2}(R)},\left\|g_{20}\right\|_{L^{2}(R)},\left\|g_{10}\right\|_{L^{\infty}(R)}$ and $\left\|g_{20}\right\|_{L^{\infty}(R)}$. 
Proof We have

$$
\begin{aligned}
& \int_{-\infty}^{\infty}\left|J_{g_{1}}(t, x)-J_{g_{2}}(t, x)\right||f(t, x)| d x \\
& \leq \frac{|m-1|}{2} \int_{-\infty}^{\infty}\left|\partial_{x} \Lambda^{-2}\left(g_{1}^{2}-g_{2}^{2}\right)\right||f(t, x)| d x \\
&+\frac{k}{2} \int_{-\infty}^{\infty} \int_{-\infty}^{\infty} e^{-|x-y|}|\operatorname{sign}(x-y)|\left|g_{1}-g_{2}\right||f(t, x)| d y d x \\
&= \frac{|m-1|}{4}\left|\int_{-\infty}^{\infty} \int_{-\infty}^{\infty} e^{-|x-y|}\right| \operatorname{sign}(x-y)|| g_{1}^{2}-g_{2}^{2}|d y| f(t, x)|d x| \\
&+c_{0} \int_{-\infty}^{\infty}\left|g_{1}-g_{2}\right| d y \int_{-\infty}^{\infty} e^{-|x-y|}|f(t, x)| d x \\
& \leq \frac{|m-1|}{4} \int_{-\infty}^{\infty}\left|\left(g_{1}-g_{2}\right)\left(g_{1}+g_{2}\right)\right| d y\left|\int_{-\infty}^{\infty} e^{-|x-y|}\right| f(t, x)|d x| \\
&+c_{0} \int_{-\infty}^{\infty}\left|g_{1}-g_{2}\right| d y \\
& \leq c_{0}(1+t) \int_{-\infty}^{\infty}\left|g_{1}-g_{2}\right| d y,
\end{aligned}
$$

in which we have used the Tonelli theorem and Lemma 2.4. The proof is completed.

We define $\delta(\sigma)$ to be a function which is infinitely differentiable on $(-\infty,+\infty)$ such that $\delta(\sigma) \geq 0, \delta(\sigma)=0$ for $|\sigma| \geq 1$ and $\int_{-\infty}^{\infty} \delta(\sigma) d \sigma=1$. For any number $h>0$, we let $\delta_{h}(\sigma)=$ $\frac{\delta\left(h^{-1} \sigma\right)}{h}$. Then we know that $\delta_{h}(\sigma)$ is a function in $C^{\infty}(-\infty, \infty)$ and

$$
\begin{cases}\delta_{h}(\sigma) \geq 0, & \delta_{h}(\sigma)=0 \quad \text { if }|\sigma| \geq h \\ \left|\delta_{h}(\sigma)\right| \leq \frac{c}{h}, & \int_{-\infty}^{\infty} \delta_{h}(\sigma)=1\end{cases}
$$

Assume that the function $u(x)$ is locally integrable in $(-\infty, \infty)$. We define an approximation function of $u$ as

$$
u^{h}(x)=\frac{1}{h} \int_{-\infty}^{\infty} \delta\left(\frac{x-y}{h}\right) u(y) d y, \quad h>0 .
$$

We call $x_{0}$ a Lebesgue point of the function $u(x)$ if

$$
\lim _{h \rightarrow 0} \frac{1}{h} \int_{\left|x-x_{0}\right| \leq h}\left|u(x)-u\left(x_{0}\right)\right| d x=0 .
$$

At any Lebesgue points $x_{0}$ of the function $u(x)$, we have $\lim _{h \rightarrow 0} u^{h}\left(x_{0}\right)=u\left(x_{0}\right)$. Since the set of points which are not Lebesgue points of $u(x)$ has measure zero, we get $u^{h}(x) \rightarrow u(x)$ as $h \rightarrow 0$ almost everywhere.

We introduce notation connected with the concept of a characteristic cone. For any $R_{0}>0$, we define $N>\max _{t \in[0, T]}\|g\|_{L^{\infty}}<\infty$. Let $\mho$ designate the cone $\left\{(t, x):|x|<R_{0}-\right.$ $\left.N t, 0 \leq t \leq T_{0}=\min \left(T, R_{0} N^{-1}\right)\right\}$. We let $S_{\tau}$ designate the cross section of the cone $\mho$ by the plane $t=\tau, \tau \in\left[0, T_{0}\right]$. 
Let $K_{r+2 \rho}=\{x:|x| \leq r+2 \rho\}$, where $r>0, \rho>0$ and $\pi_{T}=[0, T] \times R$ for an arbitrary $T>0$. The space of all infinitely differentiable functions $f(t, x)$ with compact support in $[0, T] \times R$ is denoted by $C_{0}^{\infty}\left(\pi_{T}\right)$.

Lemma 2.7 ([20]) Let the function $u(t, x)$ be bounded and measurable in cylinder $\Omega_{T}=$ $[0, T] \times K_{r}$. Iffor $\rho \in(0, \min [r, T])$ and any number $h \in(0, \rho)$, then the function

$$
V_{h}=\frac{1}{h^{2}} \iiint \int_{\left|\frac{t-\tau}{2}\right| \leq h, \rho \leq \frac{t+\tau}{2} \leq T-\rho,\left|\frac{x-y}{2}\right| \leq h,\left|\frac{x+y}{2}\right| \leq r-\rho}|u(t, x)-u(\tau, y)| d x d t d y d \tau
$$

satisfies $\lim _{h \rightarrow 0} V_{h}=0$.

Lemma 2.8 ([20]) Let $\left|\frac{\partial G(u)}{\partial u}\right|$ be bounded. Then the function

$$
H(u, v)=\operatorname{sign}(u-v)(G(u)-G(v))
$$

satisfies the Lipschitz condition in $u$ and $v$, respectively.

Lemma 2.9 Let $g$ be the strong solution of problem (7), $f(t, x) \in C_{0}^{\infty}\left(\pi_{T}\right)$ and $f(0, x)=0$.

Then

$$
\iint_{\pi_{T}}\left\{|g-k| f_{t}+\operatorname{sign}(g-k) \frac{1}{2}\left[g^{2}-k^{2}\right] f_{x}-\operatorname{sign}(g-k) J_{g}(t, x) f\right\} d x d t=0,
$$

where $k$ is an arbitrary constant.

Proof Let $\Phi(g)$ be an arbitrary twice smooth function on the line $-\infty<g<\infty$. We multiply the first equation of problem (7) by the function $\Phi^{\prime}(g) f(t, x)$, where $f(t, x) \in C_{0}^{\infty}\left(\pi_{T}\right)$. Integrating over $\pi_{T}$ and transferring the derivatives with respect to $t$ and $x$ to the test function $f$, for any constant $k$, we obtain

$$
\iint_{\pi_{T}}\left\{\Phi(g) f_{t}+\left[\int_{k}^{g} \Phi^{\prime}(z) z d z\right] f_{x}-\Phi^{\prime}(g) J_{g}(t, x) f\right\} d x d t=0,
$$

in which we have used $\int_{-\infty}^{\infty}\left[\int_{k}^{g} \Phi^{\prime}(z) z d z\right] f_{x} d x=-\int_{-\infty}^{\infty}\left[f \Phi^{\prime}(g) g g_{x}\right] d x$.

Integration by parts yields

$$
\begin{aligned}
\int_{-\infty}^{\infty}\left[\int_{k}^{g} \Phi^{\prime}(z) z d z\right] f_{x} d x= & \int_{-\infty}^{\infty}\left[\frac{1}{2}\left(g^{2}-k^{2}\right) \Phi^{\prime}(g)\right. \\
& \left.-\frac{1}{2} \int_{k}^{g}\left(z^{2}-k^{2}\right) \Phi^{\prime \prime}(z) d z\right] f_{x} d x
\end{aligned}
$$

Let $\Phi^{h}(g)$ be an approximation of the function $|g-k|$ and set $\Phi(g)=\Phi^{h}(g)$. Using the properties of $\operatorname{sign}(g-k),(29),(30)$ and sending $h \rightarrow 0$, we have

$$
\iint_{\pi_{T}}\left\{|g-k| f_{t}+\operatorname{sign}(g-k) \frac{1}{2}\left[g^{2}-k^{2}\right] f_{x}-\operatorname{sign}(g-k) J_{g}(t, x) f\right\} d x d t=0,
$$

which completes the proof. 
In fact, the proof of (28) can also be found in [20].

For $g_{10} \in H^{s}(R)$ and $g_{20} \in H^{s}(R)$ with $s>\frac{3}{2}$, using Lemma 2.2, we know that there exists $T>0$ such that two local strong solutions $g_{1}(t, x)$ and $g_{2}(t, x)$ of Eq. (3) satisfy

$$
g_{1}(t, x), g_{2}(t, x) \in C\left([0, T) ; H^{S}(R)\right) C^{1}\left([0, T) ; H^{s-1}(R)\right), \quad t \in[0, T) .
$$

\section{Main result}

Now, we give the main result of this work.

Theorem 3.1 Assume that $g_{1}$ and $g_{2}$ are two local strong solutions of Eq. (3) with initial data $g_{10}, g_{20} \in L^{1}(R) \cap H^{s}(R), s>\frac{3}{2}$. For $T>0$ in (32), it holds that

$$
\left\|g_{1}(t, \cdot)-g_{2}(t, \cdot)\right\|_{L^{1}(R)} \leq c e^{c t} \int_{-\infty}^{\infty}\left|g_{10}(x)-g_{20}(x)\right| d x, \quad t \in[0, T],
$$

where c depends on $\left\|g_{10}\right\|_{L^{\infty}(R)},\left\|g_{20}\right\|_{L^{\infty}(R)},\left\|g_{10}\right\|_{L^{2}(R)},\left\|g_{20}\right\|_{L^{2}(R)}$ and $T$.

Proof For arbitrary $T>0$ and $f(t, x) \in C_{0}^{\infty}\left(\pi_{T}\right)$, we assume that $f(t, x)=0$ outside the cylinder

$$
\uplus=\{(t, x)\}=[\rho, T-2 \rho] \times K_{r-2 \rho}, \quad 0<2 \rho \leq \min (T, r) .
$$

We set

$$
\eta=f\left(\frac{t+\tau}{2}, \frac{x+y}{2}\right) \delta_{h}\left(\frac{t-\tau}{2}\right) \delta_{h}\left(\frac{x-y}{2}\right)=f(\cdots) \lambda_{h}(*),
$$

where $(\cdots)=\left(\frac{t+\tau}{2}, \frac{x+y}{2}\right)$ and $(*)=\left(\frac{t-\tau}{2}, \frac{x-y}{2}\right)$. The function $\delta_{h}(\sigma)$ is defined in (26). Note that

$$
\eta_{t}+\eta_{\tau}=f_{t}(\cdots) \lambda_{h}(*), \quad \eta_{x}+\eta_{y}=f_{x}(\cdots) \lambda_{h}(*) .
$$

Using the Kruzkov device of doubling the variables [20] and Lemma 2.9, we have

$$
\begin{aligned}
& \iiint \int_{\pi_{T} \times \pi_{T}}\left\{\left|g_{1}(t, x)-g_{2}(\tau, y)\right| \eta_{t}\right. \\
& \quad+\operatorname{sign}\left(g_{1}(t, x)-g_{2}(\tau, y)\right)\left(\frac{g_{1}^{2}(t, x)}{2}-\frac{g_{2}^{2}(\tau, y)}{2}\right) \eta_{x} \\
& \left.\left.\quad-\operatorname{sign}\left(g_{1}(t, x)-g_{2}(\tau, y)\right)\right)_{g_{1}}(t, x) \eta\right\} d x d t d y d \tau=0 .
\end{aligned}
$$

Similarly, we have

$$
\begin{aligned}
& \iiint \int_{\pi_{T} \times \pi_{T}}\left\{\left|g_{2}(\tau, y)-g_{1}(t, x)\right| \eta_{\tau}\right. \\
& \quad+\operatorname{sign}\left(g_{2}(\tau, y)-g_{1}(t, x)\right)\left(\frac{g_{2}^{2}(\tau, y)}{2}-\frac{g_{1}^{2}(t, x)}{2}\right) \eta_{y} \\
& \left.\quad-\operatorname{sign}\left(g_{2}(\tau, y)-g_{1}(t, x)\right) J_{g_{2}}(\tau, y) \eta\right\} d x d t d y d \tau=0,
\end{aligned}
$$


from which we obtain

$$
\begin{aligned}
0 \leq & \iiint \int_{\pi_{T} \times \pi_{T}}\left\{\left|g_{1}(t, x)-g_{2}(\tau, y)\right|\left(\eta_{t}+\eta_{\tau}\right)\right. \\
& \left.+\operatorname{sign}\left(g_{1}(t, x)-g_{2}(\tau, y)\right)\left(\frac{g_{1}^{2}(t, x)}{2}-\frac{g_{2}^{2}(\tau, y)}{2}\right)\left(\eta_{x}+\eta_{y}\right)\right\} d x d t d y d \tau \\
& +\left|\iiint \int_{\pi_{T} \times \pi_{T}} \operatorname{sign}\left(g_{1}(t, x)-g_{2}(t, x)\right)\left(J_{g_{1}}(t, x)-J_{g_{2}}(\tau, y)\right) \eta d x d t d y d \tau\right| \\
= & I_{1}+I_{2}+\left|\iiint \int_{\pi_{T} \times \pi_{T}} I_{3} d x d t d y d \tau\right| .
\end{aligned}
$$

We will show that

$$
\begin{aligned}
0 \leq & \iint_{\pi_{T}}\left\{\left|g_{1}(t, x)-g_{2}(t, x)\right| f_{t}\right. \\
& \left.+\operatorname{sign}\left(g_{1}(t, x)-g_{2}(t, x)\right)\left(\frac{g_{1}^{2}(t, x)}{2}-\frac{g_{2}^{2}(t, x)}{2}\right) f_{x}\right\} d x d t \\
& +\left|\iint_{\pi_{T}} \operatorname{sign}\left(g_{1}(t, x)-g_{2}(t, x)\right)\left[J_{g_{1}}(t, x)-J_{g_{2}}(t, x)\right] f d x d t\right| .
\end{aligned}
$$

In fact, the first two terms in the integrand of (39) can be represented in the form

$$
A_{h}=A\left(t, x, \tau, y, g_{1}(t, x), g_{2}(\tau, y)\right) \lambda_{h}(*) \text {. }
$$

From Lemma 2.4 and the assumptions on solutions $g_{1}, g_{2}$, we have $\left\|g_{1}\right\|_{L^{\infty}}<C_{T}$ and $\left\|g_{2}\right\|_{L^{\infty}}<C_{T}$. From Lemma 2.8, we know that $A_{h}$ satisfies the Lipschitz condition in $g_{1}$ and $g_{2}$, respectively. By the choice of $\eta$, we have $A_{h}=0$ outside the region

$$
\{(t, x ; \tau, y)\}=\left\{\rho \leq \frac{t+\tau}{2} \leq T-2 \rho, \frac{|t-\tau|}{2} \leq h, \frac{|x+y|}{2} \leq r-2 \rho, \frac{|x-y|}{2} \leq h\right\}
$$

and

$$
\begin{aligned}
\iiint \int_{\pi_{T} \times \pi_{T}} A_{h} d x d t d y d \tau= & \iiint \int_{\pi_{T} \times \pi_{T}}\left[A\left(t, x, \tau, y, g_{1}(t, x), g_{2}(\tau, y)\right)\right. \\
& \left.-A\left(t, x, t, x, g_{1}(t, x), g_{2}(t, x)\right)\right] \lambda_{h}(*) d x d t d y d \tau \\
& +\iiint \int_{\pi_{T} \times \pi_{T}} A\left(t, x, t, x, g_{1}(t, x), g_{2}(t, x)\right) \lambda_{h}(*) d x d t d y d \tau \\
= & K_{11}(h)+K_{12} .
\end{aligned}
$$

Considering the estimate $|\lambda(*)| \leq \frac{c}{h^{2}}$ and the expression of function $A_{h}$, we have

$$
\begin{aligned}
\left|K_{11}(h)\right| \leq & c\left[h+\frac{1}{h^{2}}\right. \\
& \left.\times \iiint \int_{\left|\frac{t-\tau}{2}\right| \leq h, \rho \leq \frac{t+\tau}{2} \leq T-\rho,\left|\frac{x-y}{2}\right| \leq h,\left|\frac{x+y}{2}\right| \leq r-\rho}\left|g_{2}(t, x)-g_{2}(\tau, y)\right| d x d t d y d \tau\right],
\end{aligned}
$$


where the constant $c$ does not depend on $h$. Using Lemma 2.7, we obtain $K_{11}(h) \rightarrow 0$ as $h \rightarrow 0$. The integral $K_{12}$ does not depend on $h$. In fact, substituting $t=\alpha, \frac{t-\tau}{2}=\beta, x=\zeta$, $\frac{x-y}{2}=\xi$ and noting that

$$
\int_{-h}^{h} \int_{-\infty}^{\infty} \lambda_{h}(\beta, \xi) d \xi d \beta=1
$$

we have

$$
\begin{aligned}
K_{12} & =2^{2} \iint_{\pi_{T}} A_{h}\left(\alpha, \zeta, \alpha, \zeta, g_{1}(\alpha, \zeta), g_{2}(\alpha, \zeta)\right)\left\{\int_{-h}^{h} \int_{-\infty}^{\infty} \lambda_{h}(\beta, \xi) d \xi d \beta\right\} d \zeta d \alpha \\
& =4 \iint_{\pi_{T}} A\left(t, x, t, x, g_{1}(t, x), g_{2}(t, x)\right) d x d t .
\end{aligned}
$$

Hence

$$
\lim _{h \rightarrow 0} \iiint \int_{\pi_{T} \times \pi_{T}} A_{h} d x d t d y d \tau=4 \iint_{\pi_{T}} A\left(t, x, t, x, g_{1}(t, x), g_{2}(t, x)\right) d x d t .
$$

Since

$$
I_{3}=\operatorname{sign}\left(g_{1}(t, x)-g_{2}(\tau, y)\right)\left(J_{g_{1}}(t, x)-J_{g_{2}}(\tau, y)\right) f \lambda_{h}(*)=\bar{I}_{3}(t, x, \tau, y) \lambda_{h}(*)
$$

and

$$
\begin{aligned}
& \iiint \int_{\pi_{T} \times \pi_{T}} I_{3} d x d t d y d \tau \\
& =\iiint \int_{\pi_{T} \times \pi_{T}}\left[\bar{I}_{3}(t, x, \tau, y)-\bar{I}_{3}(t, x, t, x)\right] \lambda_{h}(*) d x d t d y d \tau \\
& \quad+\iiint \int_{\pi_{T} \times \pi_{T}} \bar{I}_{3}(t, x, t, x) \lambda_{h}(*) d x d t d y d \tau=K_{21}(h)+K_{22},
\end{aligned}
$$

we obtain

$$
\begin{aligned}
\left|K_{21}(h)\right| \leq & c\left(h+\frac{1}{h^{2}}\right. \\
& \left.\times \iiint \int_{\left|\frac{t-\tau}{2}\right| \leq h, \rho \leq \frac{t+\tau}{2} \leq T-\rho,\left|\frac{x-y}{2}\right| \leq h,\left|\frac{x+y}{2}\right| \leq r-\rho}\left|J_{g_{2}}(t, x)-J_{g_{2}}(\tau, y)\right| d x d t d y d \tau\right) .
\end{aligned}
$$

Using Lemma 2.7, we have $K_{21}(h) \rightarrow 0$ as $h \rightarrow 0$. Using (44), we have

$$
\begin{aligned}
K_{22} & =2^{2} \iint_{\pi_{T}} \bar{I}_{3}\left(\alpha, \zeta, \alpha, \zeta, g_{1}(\alpha, \zeta), g_{2}(\alpha, \zeta)\right)\left\{\int_{-h}^{h} \lambda_{h}(\beta, \xi) d \xi d \beta\right\} d \zeta d \alpha \\
& =4 \iint_{\pi_{T}} \bar{I}_{3}\left(t, x, t, x, g_{1}(t, x), g_{2}(t, x)\right) d x d t \\
& =4 \iint_{\pi_{T}} \operatorname{sign}\left(g_{1}(t, x)-g_{2}(t, x)\right)\left(J_{g_{1}}(t, x)-J_{g_{2}}(t, x)\right) f(t, x) d x d t .
\end{aligned}
$$

From (42), (46), (48), (49) and (50), we prove that inequality (40) holds. 
Let

$$
\mu(t)=\int_{-\infty}^{\infty}\left|g_{1}(t, x)-g_{2}(t, x)\right| d x
$$

We define

$$
\theta_{h}=\int_{-\infty}^{\sigma} \delta_{h}(\sigma) d \sigma \quad\left(\theta_{h}^{\prime}(\sigma)=\delta_{h}(\sigma) \geq 0\right)
$$

and choose two numbers $\rho$ and $\tau \in\left(0, T_{0}\right), \rho<\tau$. In (40), we choose

$$
f=\left[\theta_{h}(t-\rho)-\theta_{h}(t-\tau)\right] \chi(t, x), \quad h<\min \left(\rho, T_{0}-\tau\right),
$$

where

$$
\chi(t, x)=\chi_{\varepsilon}(t, x)=1-\theta_{\varepsilon}(|x|+N t-R+\varepsilon), \quad \varepsilon>0 .
$$

We note that the function $\chi(t, x)=0$ outside the cone $\mho$ and $f(t, x)=0$ outside the set $\uplus$. For $(t, x) \in \mho$, we have the relations

$$
0=\chi_{t}+N\left|\chi_{x}\right| \geq \chi_{t}+N \chi_{x}
$$

Applying (53)-(55) and (40), we have the inequality

$$
\begin{aligned}
0 \leq & \iint_{\pi_{T_{0}}}\left\{\left[\delta_{h}(t-\rho)-\delta_{h}(t-\tau)\right] \chi_{\varepsilon}\left|g_{1}(t, x)-g_{2}(t, x)\right|\right\} d x d t \\
& +\int_{0}^{T_{0}} \int_{-\infty}^{\infty}\left[\theta_{h}(t-\rho)-\theta_{h}(t-\tau)\right]\left|\left[J_{g_{1}}(t, x)-J_{g_{2}}(t, x)\right] \chi(t, x)\right| d x d t
\end{aligned}
$$

Using Lemma 2.6 and letting $\varepsilon \rightarrow 0$ and $R_{0} \rightarrow \infty$, we obtain

$$
\begin{aligned}
0 \leq & \int_{0}^{T_{0}}\left\{\left[\delta_{h}(t-\rho)-\delta_{h}(t-\tau)\right] \int_{-\infty}^{\infty}\left|g_{1}(t, x)-g_{2}(t, x)\right| d x\right\} d t \\
& +c_{0}\left(1+T_{0}\right) \int_{0}^{T_{0}}\left[\theta_{h}(t-\rho)-\theta_{h}(t-\tau)\right] \int_{-\infty}^{\infty}\left|g_{1}(t, x)-g_{2}(t, x)\right| d x d t .
\end{aligned}
$$

By the properties of the function $\delta_{h}(\sigma)$ for $h \leq \min \left(\rho, T_{0}-\rho\right)$, we have

$$
\begin{aligned}
\left|\int_{0}^{T_{0}} \delta_{h}(t-\rho) \mu(t) d t-\mu(\rho)\right| & =\left|\int_{0}^{T_{0}} \delta_{h}(t-\rho)\right| \mu(t)-\mu(\rho)|d t| \\
& \leq c \frac{1}{h} \int_{\rho-h}^{\rho+h}|\mu(t)-\mu(\rho)| d t \rightarrow 0 \quad \text { as } h \rightarrow 0,
\end{aligned}
$$

where $c$ is independent of $h$. Letting

$$
L(\rho)=\int_{0}^{T_{0}} \theta_{h}(t-\rho) \mu(t) d t=\int_{0}^{T_{0}} \int_{-\infty}^{t-\rho} \delta_{h}(\sigma) d \sigma \mu(t) d t,
$$


we get

$$
L^{\prime}(\rho)=-\int_{0}^{T_{0}} \delta_{h}(t-\rho) \mu(t) d t \rightarrow-\mu(\rho), \quad \text { as } h \rightarrow 0,
$$

from which we obtain

$$
L(\rho) \rightarrow L(0)-\int_{0}^{\rho} \mu(\sigma) d \sigma \quad \text { as } h \rightarrow 0 .
$$

Similarly, we have

$$
L(\tau) \rightarrow L(0)-\int_{0}^{\tau} \mu(\sigma) d \sigma \quad \text { as } h \rightarrow 0 .
$$

It follows from (61) and (62) that

$$
L(\rho)-L(\tau) \rightarrow \int_{\rho}^{\tau} \mu(\sigma) d \sigma \quad \text { as } h \rightarrow 0 .
$$

Send $\rho \rightarrow 0, \tau \rightarrow t$, and note that

$$
\begin{aligned}
\left|g_{1}(\rho, x)-g_{2}(\rho, x)\right| \leq & \left|g_{1}(\rho, x)-g_{10}(x)\right| \\
& +\left|g_{2}(\rho, x)-g_{20}(x)\right|+\left|g_{10}(x)-g_{20}(x)\right| .
\end{aligned}
$$

Thus, from (57), (58), (63)-(64), we have

$$
\begin{aligned}
\int_{-\infty}^{\infty}\left|g_{1}(t, x)-g_{2}(t, x)\right| d x \leq & \int_{-\infty}^{\infty}\left|g_{10}-g_{20}\right| d x \\
& +c_{0}\left(1+T_{0}\right) \int_{0}^{t} \int_{-\infty}^{\infty}\left|g_{1}(t, x)-g_{2}(t, x)\right| d x d t
\end{aligned}
$$

from which we complete the proof by using the Gronwall inequality.

\section{Competing interests}

The authors declare that they have no competing interests.

\section{Authors' contributions}

The article is a joint work of four authors who contributed equally to the final version of the paper. All authors read and approved the final manuscript.

\section{Acknowledgements}

This work is supported by the National Natural Science Foundation of China (11471263).

Received: 31 May 2014 Accepted: 15 September 2014 Published: 16 Oct 2014

\section{References}

1. Constantin, A, Lannes, D: The hydro-dynamical relevance of the Camassa-Holm and Degasperis-Procesi equations. Arch. Ration. Mech. Anal. 193, 165-186 (2009)

2. Lai, SY, Wu, YH: A model containing both the Camassa-Holm and Degasperis-Procesi equations. J. Math. Anal. Appl. 374, 458-469 (2011)

3. Degasperis, A, Procesi, M: Asymptotic integrability. In: Degasperis, A, Gaeta, G (eds.) Symmetry and Perturbation Theory, vol. 1, pp. 23-37. World Scientific, Singapore (1999)

4. Escher, J, Liu, Y, Yin, ZY: Global weak solutions and blow-up structure for the Degasperis-Procesi equation. J. Funct. Anal. 241, 457-485 (2006) 
5. Yin, ZY: Global weak solutions for a new periodic integrable equation with peakon solutions. J. Funct. Anal. 212, 182-194 (2004)

6. Fu, Y, Liu, Y, Qu, CZ: On the blow-up structure for the generalized periodic Camassa-Holm and Degasperis-Procesi equation. J. Funct. Anal. 262, 3125-3158 (2012)

7. Lin, ZW, Liu, Y: Stability of peakons for the Degasperis-Procesi equation. Commun. Pure Appl. Math. 62, 125-146 (2009)

8. Bressan, A, Constantin, A: Global conservative solutions of the Camassa-Holm equation. Arch. Ration. Mech. Anal. $183,215-239(2007)$

9. Bressan, A, Constantin, A: Global dissipative solutions of the Camassa-Holm equation. Anal. Appl. 5, 1-27 (2007)

10. Coclite, GM, Karlsen, KH: On the well-posedness of the Degasperis-Procesi equation. J. Funct. Anal. 223, 60-91 (2006)

11. Guo, ZG, Zhou, Y: Wave breaking and persistence properties for the dispersive rod equation. SIAM J. Math. Anal. 40 2567-2580 (2009)

12. Henry, D: Infinite propagation speed for the Degasperis-Procesi equation. J. Math. Anal. Appl. 311, 755-759 (2005)

13. Lenells, J: Traveling wave solutions of the Degasperis-Procesi equation. J. Math. Anal. Appl. 306, 72-82 (2005)

14. Li, LC: Long time behaviour for a class of low-regularity solutions of the Camassa-Holm equation. Commun. Math. Phys. 285, 265-291 (2009)

15. Lundmark, H, Szmigielski, J: Multi-peakon solutions of the Degasperis-Procesi equation. Inverse Probl. 19, 1241-1245 (2003)

16. Lai, SY, Wu, YH: The local strong and weak solutions to a generalized Novikov equation. Bound. Value Probl. 2013, 134 (2013)

17. Lai, SY, Li, N, Fan, S: The entropy weak solution to a generalized Degasperis-Procesi equation. J. Inequal. Appl. 2013, 409 (2013)

18. Ni, L, Zhou, Y: Well-posedness and persistence properties for the Novikov equation. J. Differ. Equ. 250, 3002-3021 (2011)

19. Tian, LX, Gui, GL, Liu, Y: On the well-posedness problem and the scattering problem for the Dullin-Gottwald-Holm equation. Commun. Math. Phys. 257, 667-701 (2005)

20. Kruzkov, SN: First order quasi-linear equations in several independent variables. Math. USSR Sb. 10, 217-243 (1970)

10.1186/1029-242X-2014-410

Cite this article as: Lai et al.: The stability of local strong solutions for a shallow water equation. Journal of Inequalities and Applications 2014, 2014:410

\section{Submit your manuscript to a SpringerOpen ${ }^{\circ}$ journal and benefit from:}

- Convenient online submission

Rigorous peer review

- Immediate publication on acceptance

- Open access: articles freely available online

- High visibility within the field

- Retaining the copyright to your article 